\title{
Study on J2EE-based Software Supporting Platform Component Technology
}

\author{
Lili Fan \\ Experimental cente, Jiujiang University, Jiujiang, 332005, China
}

Key words: J2EE, Supporting platform, Component, Component-based development.

\begin{abstract}
This paper studies large software component recognition mapping, component testing and component evaluation, proposes a set of development platform and key structure supporting twin-engine software, and discusses mutual relations among software functions. Besides, this paper takes e-commerce trading model for example and develops different e-commerce trading systems based on reconfigurable software development platform. This paper offers a new scheme for component-based production of business assembly software.
\end{abstract}

\section{Introduction}

Users have very high requirements for dynamic nature and adaptability of software. Besides, software demand is complex at time and space level. To better solve the contradiction between industrial growth and laggard software production, it is necessary to promote development of industrialization technology so as to continuously improve software production capacity. Traditional industrial production is a standardized parts manufacturing mode. It is a production mode for standard components. It aims to achieve assembly reuse. The experiment shows that component-based production must be conducted in order to gain industrial production of software. Hence, it is required to carry out scale economy and facilitate formation of software production standardization.

Corporate business information system is complex, and will involve each link of manpower, financial resource and materials in product research and development. Enterprise information develops to integrated application trend from simple application, which is the soul of enterprise development and must be adapted by an enterprise to develop enterprise information system. The demand may be changed according to development changes so as to change information system. But these propose very strict challenges to development of information system, because such development method may not meet the demand of information construction.

The researches on software engineering include component-based modeling and supporting tools. We should regulate component-based assembly and corresponding supporting tools, promote formation of supporting tools of component management, and continuously establish all kinds of testing and evaluation models so as to really achieve component-based assembly technology and uniformly regulate software component. Meanwhile, we should study modeling theories of business components, including entity description, decomposition and mapping etc. We also need to explore system component, business component and various hierarchical modeling methods.

There are great differences between component and module. The component can be assembled, while the module can only be reused. In current business component, it can be formed through step-by-step component technology, including phage component, operation component and data component. However, people rarely mention assembly technology. This results in the limitation of component-based assembly development. In the strict sense, the component can only be integrated. 
This paper proposes distributed component model which enhances business component. Business component assembly development can be conducted through technical component method etc. System assembly development can be carried out through business component assembly so as to gain enterprise-level assembly development.

\section{J2EE Twin-engine Component Platform Supporting C/S and B/S Software System Architecture}

\section{Reconfigurable J2EE Application Software Framework Technology}

J2EE framework pays much attention to software universality and expandability, which may result in the decrease of software system period and finally affect development quality. We compare traditional reuse technology and find that application framework attaches great importance to professional field. Besides, application framework has very strong correlation, and various systems can be gained through the framework. Of the framework structure is large enough, there will be more domain knowledge. In internal universal software production, abstract code may be used. In other words, framework reuse is in multiple applications and fields is gained through the universal code, which contributes to gaining universal and ready-made structure and finally achieving universality.

Software framework and mode are same, but they differ a lot. Design mode proposes methods to solve problems existing in the environment, and abstract framework can be manifested through the code. Meanwhile, reuse and direct execution can be achieved. However, the mode can only be expressed through examples. The mode is smaller than framework. Multiple design modes may appear in one framework, but wee can develop different applications for different modes. Generally, the system functions can be gained through various components. In general, if the software fails to reach the demand, new components can be gained through various methods. This is also beneficial to assemble new components. Thus, we may use white box and black box in system development process, but white box framework continuously develops to black box framework. The goal of system development is to achieve black box.

\section{Twin-engine Technology Supporting Software System Architecture}

We may observe single data model and promote construction of new system platform through engine platform so as to gain new application environment and achieve system reconstruction. According to application system framework and application component, application software is obtained through system mapping. The mapping engine achieves system modeling. In addition, the function of mapping engine can be exerted greatly according to system framework and application construction mode. Finally, the application software is obtained.

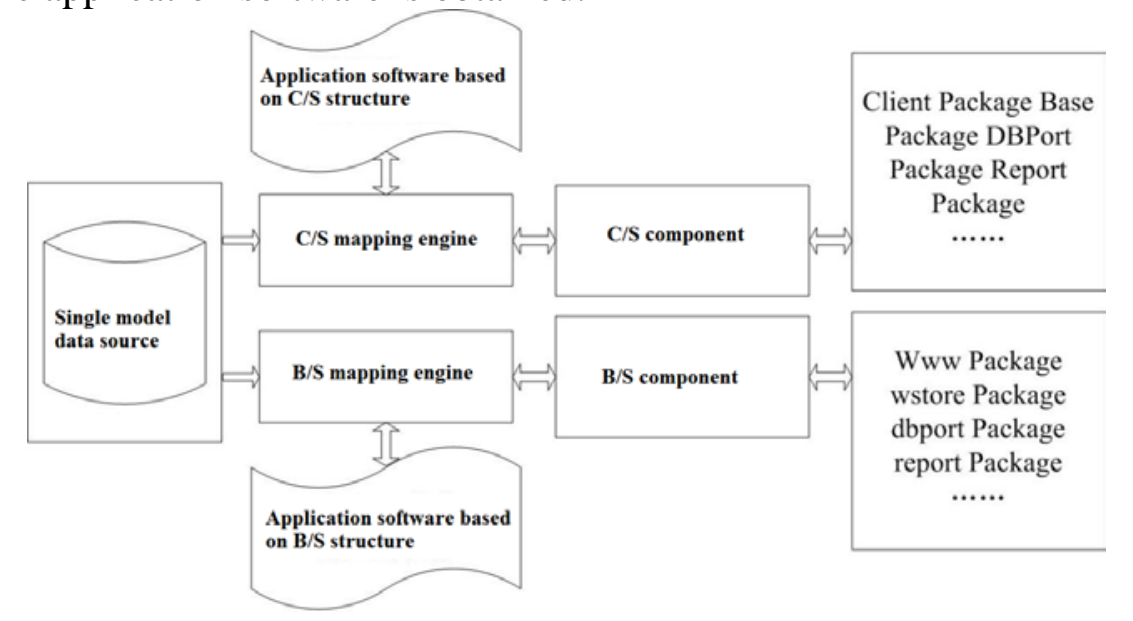


Fig. 1 Diagram of twin-engine software architecture

Language as a kind of network programming uses standard byte on virtual machine and owns an independent platform. Thus, it is the most important method for system development. In language development process, component model, computation norm and database interconnection scheme are important contents of technological development.

Application system is characterized by easy distribution and integration. Various problems may exist in system design process. As a computer application technology, existing computer system may be established in enterprise system. But existing computer system fails to achieve parallel connection, so we need to facilitate software development through computer system integration and gain different engines through gaining different view layers. If we model the view layer, different page transformation can be promoted through rendering method. This contributes to achieving fast modeling. On the other hand, it can better support the view layer.

Software development method needs to adapt information construction demand of public institutions, continuously enhance software development degree, promote software quality improvement and maintain software. This is the most important feature of software development, but software development also has the problem of theoretical research. J2EE software component technology studied in this research is a kind of information system software component technology. It is necessary to construct different software supporting platforms according to different theoretical systems under platform development, while software technology development is an important content.

The structure chart of introducing mode is as follows. In current structural system diagram, rendering engine and explaining engine exchange. This is the core issue of view work.

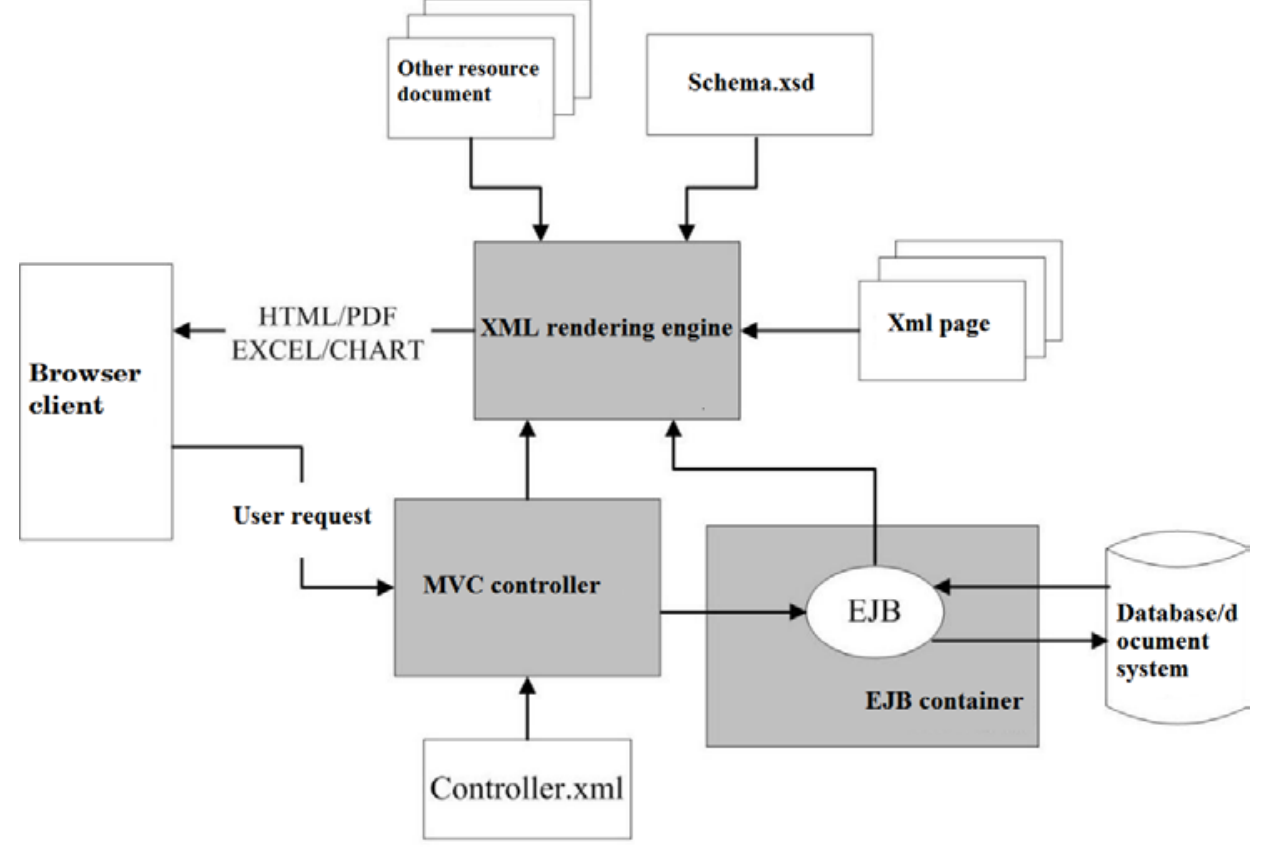

Fig. 2 MVC overall structure with XML rendering engine

\section{Component-based E-commerce Trading Model}

To promote faster development of e-commerce, we need to analyze versatility and flexibility of e-commerce due to its dynamic nature. This paper studies the development of e-commerce. E-commerce trading is quick market response of enterprises based on dynamic and diversified trading mode in order to achieve trading flexibility and trading sped. This is a new e-commerce 
trading model. Such model carries out e-commerce trading through dynamic form and can respond e-commerce trading mode and rules. The strategy can be adjusted in time to gain competition advantage.

\section{Dynamic E-commerce Trading Process Modeling}

We need to express different trading processes and different trading modes through abstract method, which can be independent of business process. Besides, business process configuration can be conducted. Some business processes are optional, and some modes are optional. Users can decide where there us choice necessity according to different modes. But we need to execute business process according to strict sequence, because these business processes can be conducted at the same time. 3 stages will be generated in education process, including preprocessing stage, trading stage and postprocessing stage. The business process is as shown in the following figure:

$$
\begin{aligned}
& \text { AeBProcParaS }=\{\text { PreTransProc U PTransProc U PostTransProc }\} \\
& =\{(\text { PreTransPequred } \cup \text { PreTransOpational }) \cup(\text { PTransRequred } U \\
& \text { PTransOpational }) \cup(\text { PostTransRequred U PostTransOpational })\}
\end{aligned}
$$

\section{E-commerce System reconfiguration}

When the system operates, we can satisfy different customer needs and achieve system construction. Construction process and construction application are same. We may alter system data layer model through database and view. Besides, we may change the model at application layer. Through creating different buttons, we can change and modify contents. Setting window and page window can be gained through modifying the model. The model at presentation layer mainly modifies display attribute of application system. it better achieves mapping function ands contributes to new system generation and new process reconstruction.

\section{Conclusions}

This paper studies J2EE-based software supporting platform component technology, proposes the design thought of twin-engine software platform and designs reconfigurable software development platform which supports twin-engine software system structure and its key business component. Based on cast study of e-commerce trading system, this paper studies main business process and business mode of e-commerce trading system and e-commerce trading system in circulation field, and designs general framework of e-commerce trading system. we may develop commerce trading system adapting agricultural product circulation for different software development platforms.

\section{References}

[1] Wang Qiangbing, Liu Guangzhong, J2EE-based web enterprise calculation. Computer Engineering, 2012, 25(1):262-264

[2] Lin QI, Advanced programming of J2EE Web application. Beijing: Tsinghua University Press, 2012

[3] Jiang Xiaoyi, Liu Zhongbing, Xu Jiajing, JSP database development examples. Beijing: Electronic Industry Press, 2015

[4] Chen Liying, JMS development technology of PTP information communication. High Performance Computing Technology, 2015, 39(7): 45-50 
[5] Zhang Jian et al., EJB-based component development technology. Computer Engineering, 2012, 28(3):89-91 